\title{
Caracterización de los depósitos secundarios de rodados fluviales del río Uruguay inferior: su aplicación en contextos arqueológicos de las llanuras interiores del nordeste argentino
}

\section{Characteristcs of secondary lithic deposits deposits at the lower Uruguay River: applications for archaeological contexts in the inland plains of northeastern Argentina}

\author{
Eduardo Apolinaire \\ Facultad de Ciencias Naturales y Museo. Universidad Nacional de La Plata. La Plata, Buenos Aires, Argentina
}

Resumen:En este trabajo se presentan los estudios de campo y laboratorio llevados a cabo sobre gravas recolectadas en el curso inferior del río Uruguay. El objetivo planteado es brindar una caracterización desde un punto de vista arqueológico de los depósitos secundarios con rocas aptas para la manufactura de artefactos líticos que se extienden en la margen derecha del río, entre las localidades de Concordia y Puerto Campichuelo. Con este fin se relevaron diversas características de los rodados y se cuantificó la variación geográfica de estas variables, caracterizando los depósitos según su ubicación longitudinal en el curso fluvial. Esta caracterización es relevante para la comparación de los depósitos naturales con las particularidades de las gravas recuperadas en los sitios arqueológicos a lo largo de la provincia de Entre Ríos y áreas circunvecinas. Los datos generados fueron utilizados para interpretar las posibles áreas de aprovisionamiento de materias primas de un conjunto de sitios arqueológicos ubicados en las llanuras interiores entrerrianas.

Palabras-clave: Rodados fluviales. Depósitos de gravas. Base regional de recursos líticos. Llanuras interiores. Entre Ríos.

\begin{abstract}
In this paper, we present field and laboratory studies addressing gravel deposits of the lower Uruguay River. The goal was to describe the secondary deposits of pebbles located on the right bank of the river, between Concordia and Puerto Campichuelo, from an archaeological point of view. To do so, we considered various features of the surroundings and their geographical variations to describe these deposits according to their position along the course of the river. This information is important for the comparision of natural deposits with the features of pebbles recovered in regional archaeological contexts. The resulting data was used to better understand the strategies of provisioning raw material implemented by the groups that inhabited the inland plains of the province of Entre Ríos.
\end{abstract}

Keywords: Fluvial pebbles. Gravel deposits. Regional base of lithic resources. Inland plains. Entre Ríos province.

APOLINAIRE, Eduardo. Caracterización de los depósitos secundarios de rodados fluviales del río Uruguay inferior. Su aplicación em contextos arqueológicos de las llanuras interiores del nordeste argentino. Boletim do Museu Paraense Emílio Goeldi. Ciências Humanas, v. 13, n. 2, p. 443-457, maio-ago. 2018. DOI: http://dx.doi.org/10.1590/1981.81222018000200010.

Autor para correspondência: Eduardo Apolinaire. Universidad Nacional de La Plata. Paseo del Bosque s/n, La Plata 1900, Buenos Aires, Argentina (eapolinaire@gmail.com). ORCID: http://orcid.org/0000-0002-3718-9228.

Recebido em 26/10/2017

Aprovado em 25/04/2018
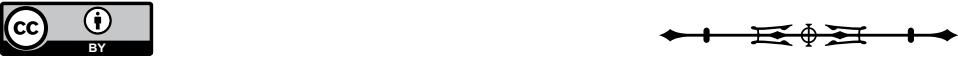


\section{INTRODUCCIÓN}

A partir de la década de 1990 se observa en la bibliografía arqueológica argentina un aumento de los trabajos orientados a caracterizar la estructura regional de recursos líticos (Nami, 1992; Berón et al., 1995; Franco; Borrero, 1999; Bayón et al., 2006; Berón, 2006). En los últimos años esta tendencia se ha profundizado, lo que ha generado detallados conjuntos de datos sobre diversas materias primas líticas, los cuales fueron construidos en función de objetivos, problemáticas y escalas espaciales de índole arqueológica (Carballido Calatayud; Pereyra, 2012; Colombo, 2013; Hermo et al., 2013; Bonomo; Prates, 2014; Banegas et al., 2015; Bobillo; Hocsman, 2015; Heider et al., 2015; Skarbun, 2015; Turnes et al., 2016). Estos trabajos se han centrado en el estudio del abastecimiento lítico tanto en afloramientos primarios de roca como en depósitos secundarios de rodados, y han servido para el estudio de diversos aspectos de los grupos humanos, tales como movilidad, patrón de asentamiento, elecciones tecnológicas, técnicas de talla, intercambio de materia prima, entre otros.

En la provincia de Entre Ríos, el aprovisionamiento lítico ha sido un tópico que comenzó a abordarse con profundidad sólo a partir de los estudios sistemáticos realizados en los últimos años (Bonomo; Blasi, 2010; Hocsman, 2015; Martínez et al., 2015; Apolinaire et al., 2016). En este sentido, es aún escasa la información sobre la disponibilidad, características petrográficas y localización geográfica específica de los depósitos de materias primas líticas a escala regional. Debido a esto se torna necesario continuar y ampliar los estudios tendientes a definir la oferta ambiental de rocas y su estructuración espacial, es decir, la base regional de recursos líticos (BRRL) $)^{1}$ del Nordeste argentino. A los fines de aportar a dicho objetivo general, este trabajo pretende caracterizar la estructura y disponibilidad de los depósitos secundarios de rocas que se encuentran a lo largo del curso del río Uruguay en la provincia de Entre Ríos. Estos depósitos han sido recurrentemente mencionados en la bibliografía regional como fuentes de abastecimiento de rocas aptas para la manufactura de artefactos (Bonomo; Blasi, 2010; Buc; Silvestre, 2011; Castro, 2013; Apolinaire et al., 2016). Sin embargo, aún no se cuenta con estudios detallados que permitan una mayor precisión a la hora de establecer las elecciones tecnológicas de los grupos que aprovecharon estos depósitos, las cuales fueron necesariamente condicionadas por la disponibilidad y estructura interna de los mismos.

Como caso de estudio, se aborda aquí el conjunto de sitios arqueológicos ubicados en las llanuras interiores del departamento Gualeguay. En estudios previos (Apolinaire et al., 2016; Apolinaire, 2017) se describieron los materiales líticos recuperados en varios de estos sitios arqueológicos, muchos de los cuales son producto de la talla de rodados fluviales. A partir de la delimitación de las características de los depósitos de rodados del río Uruguay en cuanto a tamaño, morfología y petrografía se busca precisar y delimitar las posibles áreas de procedencia de estos conjuntos líticos.

\section{LOS DEPÓSITOS DE GRAVAS DEL RÍO URUGUAY}

La cuenca del río Uruguay drena una amplia área que incluye el sudeste de Brasil, noreste de Argentina y oeste de Uruguay. Este río presenta un régimen irregular y variable a lo largo de su curso debido a la presencia de importantes afluentes cuyos aportes se ubican en diferentes sectores de su recorrido (e.g. Pelotas, Ijuí, Ibicuí, Negro, Gualeguaychú) (Iriondo; Kröhling, 2008). El área drenada por el río Uruguay ha sido dividida en tres sectores. Los dos primeros comprenden la cuenca alta y media, desde las nacientes del río en la Sierra Geral hasta los saltos cercanos a localidad de Concordia en Entre Ríos. A partir de dicha localidad y hasta su desembocadura en el Río de la Plata, se constituye la cuenca baja. Es este último sector el que compone el área de estudio considerada en este trabajo.

Dentro del territorio argentino, el río Uruguay disecta secuencias estratigráficas compuestas por una gran variedad

\footnotetext{
Sensu Ericson (1984)
}

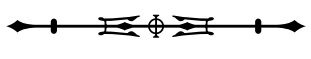


de formaciones geológicas que en muchos casos presentan facies diagenizadas. El transporte de clastos de las rocas de estas formaciones ha generado depósitos de gravas de variable litología, tamaño y morfología. En el tramo inferior del río los depósitos de gravas han sido asignados a dos formaciones geológicas diferentes, la Formación El Palmar y la Formación Concordia (Aceñolaza, 2007).

La Formación El Palmar (Iriondo, 1980), también llamada Fm. Salto Chico (Gentile; Rimoldi, 1979) o Fm. Salto (Goso; Bossi, 1966) en la República Oriental del Uruguay (ROU), corresponde a una antigua llanura aluvial (actual terraza alta del río Uruguay), formada por un paleocauce que presentaba un patrón sinuoso con grandes meandros. Esta terraza presenta un desarrollo variable a lo largo de la margen argentina del cauce, alcanzando $15 \mathrm{~km}$ de ancho entre las localidades de San José y Ubajay. La superficie de la terraza es disecada por afluentes locales que han generado sus propios valles fluviales, convirtiendo la terraza alta en un paisaje de colinas. A partir del amplio contenido fósil recuperado, que incluye mamíferos de edad Lujanense, se ha inferido que la Fm. El Palmar se habría depositado durante el Pleistoceno (Tonni, 1987; Noriega et al., 2004; Ferrero et al., 2007), lo cual también es sostenido por las dataciones por termoluminiscencia (Kröhling, 2009).

Esta formación corresponde principalmente a depósitos conglomerádicos del río Uruguay. Estos están compuestos por arenas amarillentas a rojizas con estratos lenticulares de cantos rodados y gravas de composición silícea y, en algunos sectores, presenta intercalaciones de bancos de areniscas silicificadas. Los estratos de cantos rodados forman los denominados 'cerritos' del río Uruguay, que son relieves positivos en la terraza fluvial que perduraron gracias a la mayor resistencia a la erosión de estas lentes (Kröhling, 2009; Veroslavsky; Ubilla, 2007). Esta unidad apoya en discordancia erosiva sobre los basaltos de la Fm. Serra Geral o sobre las areniscas de la Fm. Puerto Yerúa y está cubierta por sedimentos eólicos del Holoceno tardío (Aceñolaza, 2007; Iriondo; Kröhling, 2008).
Kröhling (2009) señala que, entre las localidades de Federación y Concepción del Uruguay, la Fm. El Palmar comprende fundamentalmente depósitos de arenas gruesas y medias con estratificación planar. Intercalados en estos depósitos, se pueden encontrar lentes de rodados (calcedonia, cuarzo, arenisca y, en menor medida, basalto), de gran extensión y pocos metros de potencia, que corresponderían a eventos de alta energía. El tamaño de los rodados disminuiría de norte a sur desde grueso a fino, al tiempo que aumentaría la redondez de forma correlativa. Al sur de la localidad de Concepción del Uruguay, habría existido un importante paleo-relieve en la Fm. Fray Bentos que evitó la depositación de la Fm. El Palmar (Iriondo; Kröhling, 2008).

Los depósitos sedimentarios de la terraza baja del río Uruguay han sido definidos por Iriondo y Kröhling (2008) como Formación Concordia. Esta unidad se desarrolló durante el Holoceno y está compuesta por dos miembros de similares características sedimentarias, divididos por un paleosuelo generado durante el máximo climático del Holoceno medio. Estos miembros se componen de sedimentos franco arenosos y areno arcillosos de color marrón grisáceo a marrón rojizo, masivo o groseramente estratificado. Ambos miembros se habrían depositado como facies de inundación durante dos períodos semiáridos a los cuales se intercaló una fase húmeda. El miembro inferior se habría depositado durante el Holoceno temprano mientras que el superior sería posterior a los 1000 años AP (Iriondo, 2010).

En la provincia de Entre Ríos el perfil tipo de esta formación se encuentra en la localidad de Concordia, donde el miembro inferior se compone de base a techo por arenas arcillosas con lentes de guijas silíceas y basálticas subredondeadas a redondeadas seguidas de arcillas cohesivas y un suelo enterrado en la parte superior. El miembro superior se compone de arenas cuarzosas muy finas seguidas de limos arcillosos con gran cantidad de arena muy fina a fina y un suelo desarrollado durante el Período Cálido Medieval (Iriondo; Krohling, 2008).

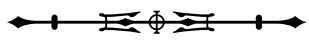




\section{METODOLOGÍA}

El área de estudio considerada para el muestreo de rodados fluviales se circunscribe a la margen derecha del río Uruguay, entre la represa de Salto Grande (Departamento Concordia) al norte y Puerto Campichuelo (Departamento Uruguay) al sur. La ubicación de las unidades de muestreo es sintetizada en la Figura 1. La recolección de los clastos líticos se llevó a cabo mediante el planteo de 23 unidades cuadradas de muestreo de $50 \mathrm{~cm}$ de lado ubicadas en zonas accesibles de la costa del río Uruguay (Figura 1). Durante las tareas de campo se recolectaron todos los clastos de tamaño sábulo o mayores que se ubicaron en cada unidad de muestreo. De esta forma, el tamaño de muestra supera ampliamente el tamaño de muestra mínimo para obtener una muestra significativa a los fines planteados. El número mínimo de clastos (n) a recolectar por cada unidad de muestreo puede establecerse asumiendo que la población de clastos tiende al infinito $(N=\infty)$ y que la estimación de los parámetros puede presentar un error aceptable del 20\% respecto al valor poblacional (error estándar relativo $=0,2)$. Considerando estos valores y un nivel de confianza del 95\% ( $p<0,05)$ el tamaño mínimo para cada unidad de muestreo es de 25 clastos (Sheenan, 1988; Zar, 2010).

Es interesante señalar que debieron descartarse durante los trabajos de campo numerosas zonas factibles de ser muestreadas. Esto se debe a que en muchas de ellas se encuentran canteras y sectores de lavado de arena y rodados que depositan en las zonas de playa el descarte de su producción alterando las características naturales de las playas. En este sentido, debido a la mayor cantidad de establecimientos de este tipo en sectores meridionales del cauce, las zonas muestreadas en estas áreas son menores que en los sectores más septentrionales del cauce.

Posteriormente a su recolección, los conjuntos de rodados fueron analizados en laboratorio. Para el desarrollo de estos estudios se siguieron los lineamientos planteados en diversos trabajos arqueológicos(Nami, 1992; Berón etal., 1995;
Franco; Borrero, 1999; Bonomo; Prates, 2014; Turnes et al., 2016). Se registraron las siguientes variables para cada clasto: dimensión de ejes ortogonales mayor, intermedio y menor, redondez, esfericidad y morfología (Zingg, 1935). Cabe destacar que no se consideraron para el análisis los rodados pequeños (cuyo eje mayor presenta dimensiones inferiores a $3 \mathrm{~cm}$ ) ya que estas son las dimensiones mínimas requeridas para la talla (Franco; Borrero, 1999).

La morfología de los clastos disponibles en los depósitos secundarios es significativa para poder abordar las elecciones técnicas vinculadas al aprovisionamiento lítico y los modos de talla. Los clastos discoideos (laminares y discoidales) resultan útiles para la realización de instrumentos sobre núcleo, ya sea unifaciales o bifaciales, a través de la modificación de sus bordes y pueden ser tallados por percusión directa a mano alzada. En contraposición, los clastos esféricos y cilíndricos, al no presentar caras planas utilizables como plataformas de percusión, suelen ser trabajados mediante el empleo de la técnica de talla bipolar. Esta técnica, que consiste en la aplicación de una fuerza compresiva sobre un clasto apoyado en un soporte que actúa como yunque, genera una fractura útil que puede ser usada como plataforma de percusión (Cotterell; Kamminga, 1987). El tamaño de los rodados también puede funcionar como un importante criterio de selección ya que el volumen inicial de la roca influye en el posterior tamaño y tipo de herramienta a manufacturar. El tamaño de los clastos disponibles a lo largo de un curso fluvial depende de la capacidad de carga del río. Por lo tanto, es posible delimitar las áreas de aprovisionamiento más probables de los nódulos presentes en los sitios arqueológicos a partir de la comparación de sus dimensiones con los rangos de tamaño de clastos naturales disponibles a lo largo del río.

Finalmente, se seleccionaron al azar 30 clastos de cada unidad de muestreo, los cuales fueron fracturados a los fines de definir la roca que los compone a partir de un corte fresco. La determinación de las rocas se realizó de modo macroscópico y con ayuda de lupa binocular. También fue relevado el color de los rodados, tanto de la superficie

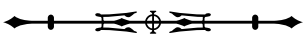



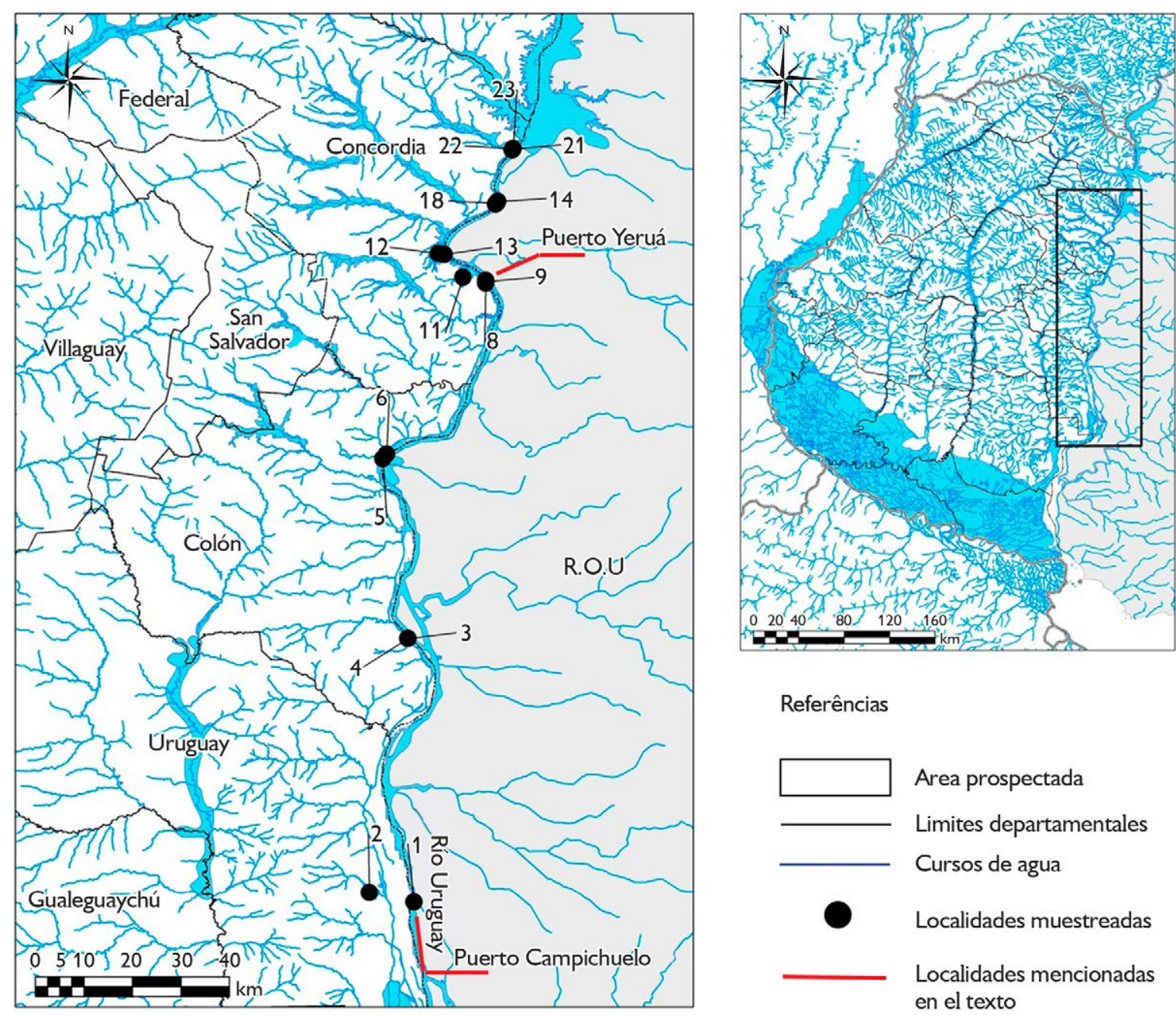

\section{Referências}

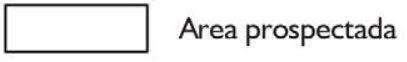

Limites departamentales

Cursos de agua

Localidades muestreadas

Localidades mencionadas en el texto
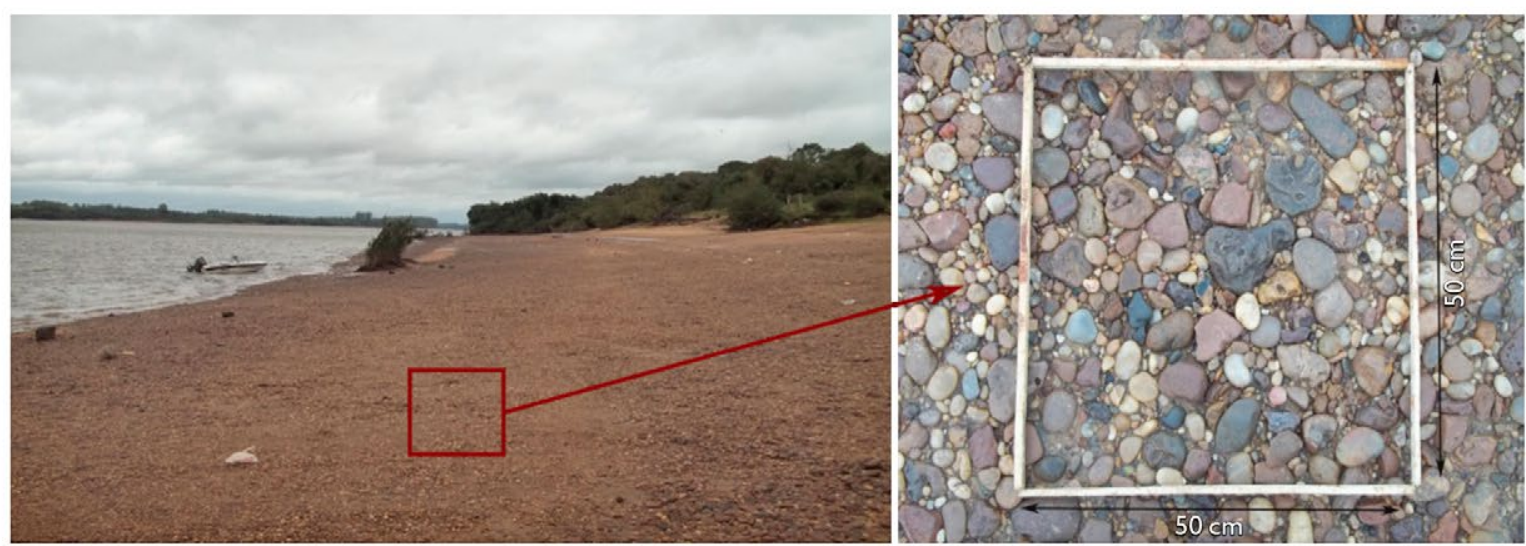

Figura 1. Depósitos secundarios relevados en la cuenca del río Uruguay. Elaborado por Eduardo Apolinaire (2017).

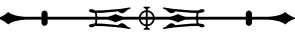


externa (corteza) como el color predominante de la matriz interna en el caso de los rodados fracturados. Esta variable ha sido considerada en muchos casos como una variable relevante para comprender de un modo más completo el conjunto de factores que afectan las elecciones vinculadas al aprovisionamiento de las rocas (Flegenheimer; Bayón, 1999; Jones; McGregor, 2002; Taçon, 2008; Colombo; Flegenheimer, 2013; Apolinaire, 2017).

\section{RESULTADOS}

La muestra analizada comprende un total de 1.542 rodados fluviales provenientes de 23 unidades de muestreo. La determinación de las materias primas se realizó a partir de un total de 690 rodados que fueron seleccionados al azar para su fractura. Los valores máximos, la media y el desvío estándar de las dimensiones de estas gravas son sintetizadas en la Tabla 1.

Para abordar la disponibilidad de clastos según tamaño a lo largo del río se realizó una correlación entre las dimensiones medias de los rodados (eje mayor y volumen) de cada unidad de muestreo y su ubicación a lo largo del río Uruguay. Para establecer esta posición se midió la distancia entre cada unidad de muestreo y un punto de referencia ubicado aguas arriba, coincidente con la división entre los sectores medio e inferior del curso del río Uruguay: el borde sur del Lago de Salto Grande (Departamento Concordia). Se observó una fuerte correlación negativa (rho $=-0.727 ; p<0.01$ ) entre la distancia y el eje mayor de los rodados, indicando que a medida que aumenta la distancia de transporte de los rodados su tamaño disminuye (Figura 2A). De hecho, los rodados disponibles en el sur del río Uruguay no suelen superar los $5 \mathrm{~cm}$ de largo. De modo similar, el volumen de los rodados disminuye a medida que aumenta su distancia de transporte $(r h o=-0.685 ; \mathrm{p}$ $<0.01$ ) (Figura 2B). Es decir, los rodados con volúmenes mayores $(>10 \mathrm{~cm}$ ) se encuentran al norte del curso del río Uruguay dentro de la provincia de Entre Ríos.

La morfología más abundante en los depósitos naturales respecto a la muestra total es la discoidal $(36 \%, n=565)$,
Tabla 1. Dimensiones y peso de los rodados fluviales analizados. Elaborado por Eduardo Apolinaire (2017).

\begin{tabular}{c|c|c|c}
\hline & Máximo & Media & Desvío Estándar \\
\hline Largo $(\mathrm{mm})$ & 170 & 47,12 & 19,53 \\
\hline Ancho $(\mathrm{mm})$ & 110 & 33,10 & 14,32 \\
\hline Espesor $(\mathrm{mm})$ & 82 & 20,66 & 9,56 \\
\hline Peso $(\mathrm{g})$ & 1.457 & 61,85 & 114,71 \\
\hline Volumen $\left(\mathrm{cm}^{3}\right)$ & $1.307,90$ & 50,81 & 95,68 \\
\hline
\end{tabular}

seguida por la esférica (23\%, $n=359)$, la cilíndrica $(21 \%$, $n=325)$ y la laminar (19\%, $n=293)$. Se observan ciertas diferencias en la morfología de las gravas a lo largo de los depósitos, si bien no pudieron identificarse tendencias claras (Figura 3). La morfología cilíndrica disminuye significativamente en el tramo más meridional (120-160 km). Asimismo, en los tramos más septentrionales (0-80 km), la morfología laminar presenta frecuencias relativas menores. Por último, la morfología discoidal tiende a disminuir con la distancia, excepto en el último tramo, mientras que la morfología esférica mantiene valores relativamente semejantes.

En cuanto a las rocas representadas en los depósitos secundarios, los rodados de calcedonia son los más abundantes (32,1\%, $n=222$ ), seguidos por el cuarzo $(16,8 \%, n=116)$, la arenisca $(13,6 \%, n=94)$ y la caliza silicificada (12,6\%, $n=87)$. También se han identificado otras rocas en menor frecuencia como los basaltos $(7,3 \%$, $n=53)$, cuarcitas (6,6\%, $n=46)$, riolitas (5,3\%, $n=37)$, xilópalos $(0,5 \%, n=4)$ y otras rocas que no pudieron identificarse $(4,7 \%, n=33)$ (Figura 4A). Es interesante considerar la gran variabilidad registrada en calidad para la talla de estas rocas. La presencia de diferentes clases de cementos, venillas, fracturas, inclusiones minerales, tamaños de grano y tipo de fractura llevaron a identificar rocas de buena, regular y mala calidad en todos los tipos de materia prima relevada. Una excepción a esto son los rodados de cuarzo cuya calidad para la talla está fuertemente condicionada por la fractura recta de este mineral.

Respecto al color de las materias primas, predomina el color castaño $(25 \%, n=178)$, seguido por el

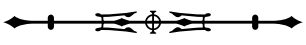




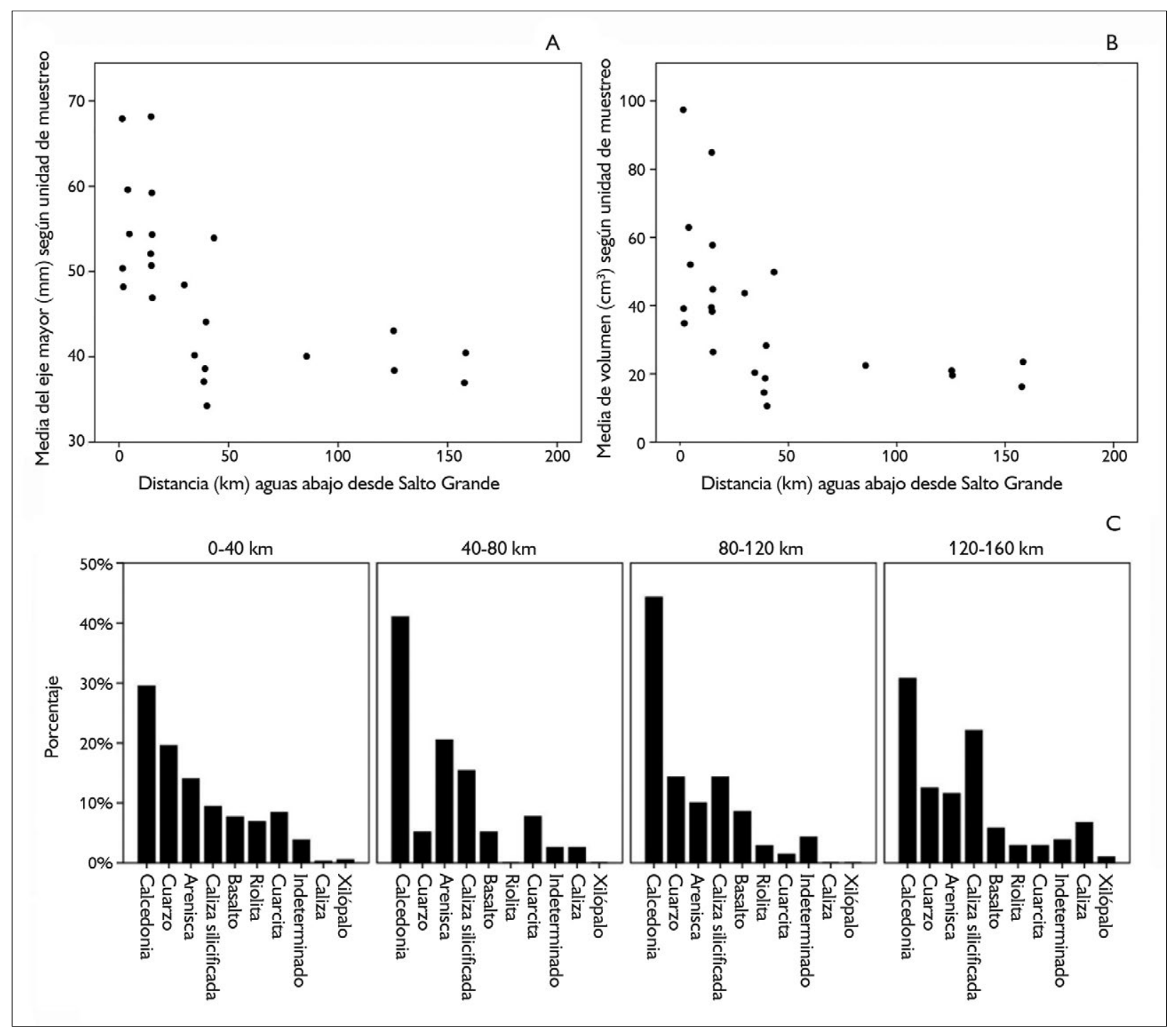

Figura 2. Distribución del largo, volumen y materia prima de los clastos según su posición sobre el cauce principal del río Uruguay: A) eje mayor medio de cada depósito según distancia de transporte; B) volumen medio de cada depósito según distancia de transporte; C) distribución de porcentajes de clases de materia prima según distancia de transporte. Elaborado por Eduardo Apolinaire (2017).

transparente $(23,64 \%, n=163)$. Los colores rojizo $(14 \%$, $\mathrm{n}=97)$, gris $(11,51 \%, \mathrm{n}=79)$ y blanco $(11 \%, \mathrm{n}=79)$ se registraron en porcentajes menores, mientras que el amarillo (4\%, $n=33)$, negro (3\%, $n=23)$, verde $(2 \%, n=18)$ y anaranjado $(2 \%, n=18)$ se encuentran escasamente representados (Figura 4B). No se observó una asociación entre los colores y las clases de materia prima registrada.
Al considerar las clases de materia prima a lo largo del río Uruguay, tomando como referencia la distancia a Salto Grande, se observa que las distintas materias primas no se presentan con frecuencias semejantes (Figura 2C). Si bien los depósitos a lo largo de todo el río Uruguay presentan un predominio de clastos de calcedonia, su frecuencia relativa varía según la categoría de distancia considerada, constituyendo más del $40 \%$ de las gravas en

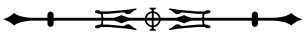


los depósitos ubicados entre 40 y 120 km del salto. Los clastos de cuarzo y arenisca, por otra parte, se presentan de modo relativamente uniforme a lo largo de los depósitos, a excepción del tramo 40-80 km, donde el cuarzo es escaso y aumenta el porcentaje de arenisca. En cuanto a la caliza silicificada, es interesante destacar que su frecuencia relativa aumenta a partir de los $40 \mathrm{~km}$ río abajo desde el Salto Grande del río Uruguay. Es posible que esto se deba a que sus afloramientos primarios se ubican al sur de la localidad de Puerto Yerúa y, por lo tanto, su aporte a la carga de gravas del río es más relevante aguas abajo de esta localidad. Las restantes rocas no presentan una variación significativa, si bien es posible que esto esté enmascarado por su baja frecuencia relativa respecto al total de cada conjunto. Es interesante señalar al respecto el caso de las cuarcitas, que presentan una escasa representación en los sectores más meridionales del curso del río Uruguay. Estas rocas provienen de la Fm. Botucatú y de intercalaciones detríticas de la Fm. Serra Geral que afloran al norte del salto y, por esta razón, es esperable que presenten mayores frecuencias en los depósitos septentrionales.

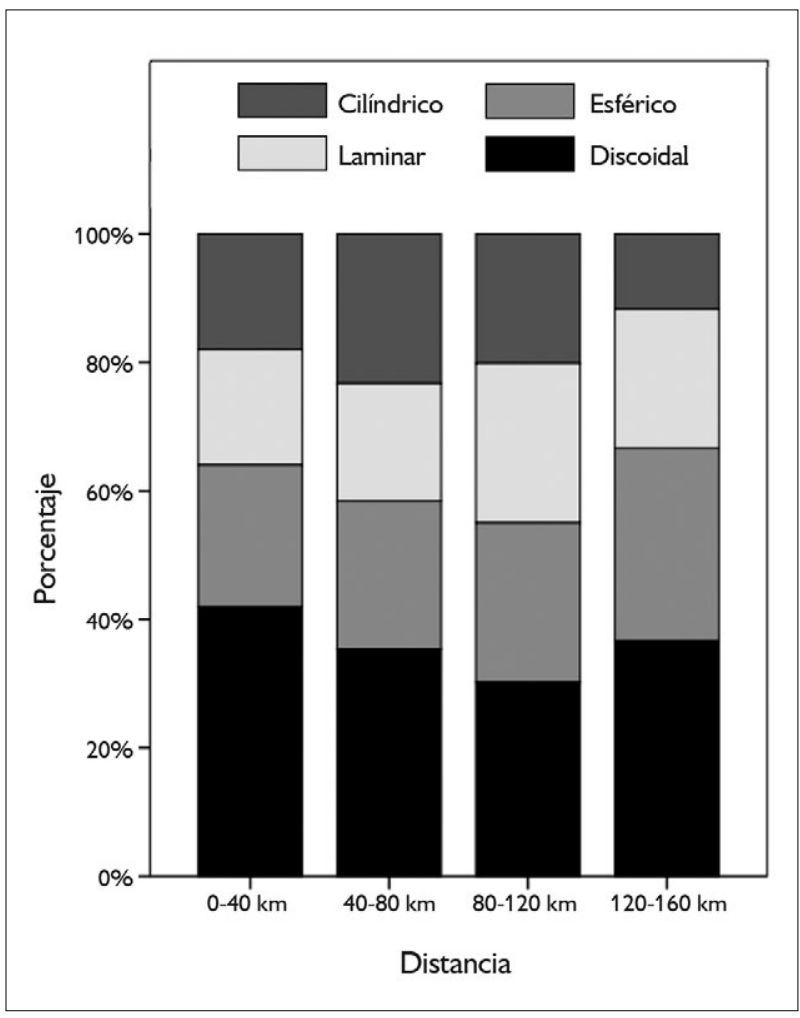

Figura 3. Morfología de los rodados según distancia de transporte. Elaborado por Eduardo Apolinaire (2017).

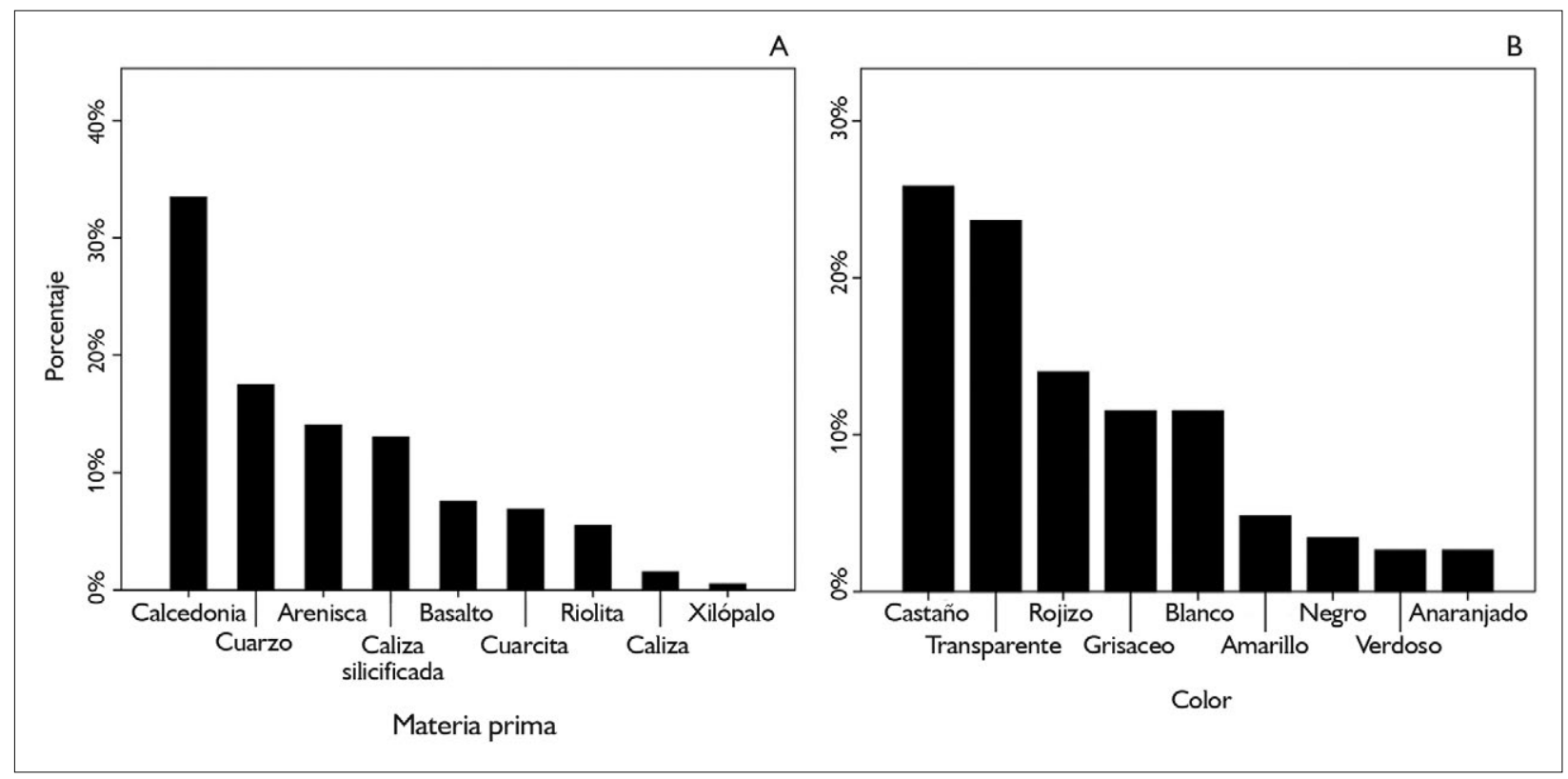

Figura 4. Materia prima y color interno en los depósitos de rodados del río Uruguay inferior: A) frecuencia percentual de clases de materia prima; B) frecuencia percentual de clases de color. Elaborado por Eduardo Apolinaire (2017).

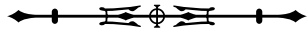




\section{CASO DE ESTUDIO: LAS LLANURAS INTERIORES ENTRERRIANAS}

El sudoeste del actual territorio de Entre Ríos es un sector donde las materias primas útiles para la manufactura de artefactos líticos son muy escasas (Tabla 2). Durante diversas tareas de prospección arqueológica fue posible detectar en este sector 19 sitios arqueológicos, 14 de los cuales presentan contextos con materiales líticos (Figura 5). Los estudios tecnológicos sobre estos materiales han sido presentados en trabajos previos (Apolinaire et al., 2016; Apolinaire, 2017) y sólo se abordarán aquí aquellos aspectos útiles para los análisis de procedencia.

Los conjuntos presentan una gran diversidad de materias primas, tanto locales como alóctonas, donde las areniscas, calcedonias y calizas silicificadas son las rocas más frecuentemente aprovechadas. Otro hecho a tener en cuenta es la variación en la representación de materias primas entre los sitios de acuerdo a un eje norte-sur.
La mayoría de los sitios más septentrionales, tienen a la arenisca como roca mayoritaria, mientras que en los sitios ubicados al sur predominan materias primas procedentes de rodados fluviales como la calcedonia o la caliza silicificada (Apolinaire et al., 2016; Apolinaire, 2017).

En general, la evidencia recuperada en los sitios arqueológicos del departamento Gualeguay indica que esta variedad de materias primas se utilizó para la elaboración, ya sea por talla (Figura 5A y 5B) o por picado, pulido y abrasión (Figura 5C) de diversos artefactos líticos que habrían sido utilizados en actividades tales como el procesamiento de carcasas, la molienda de sustancias vegetales y minerales y la manufactura de alfarería (Apolinaire et al., 2016; Bastourre; Apolinaire, 2017). Si bien el carácter reducido de la muestra no ha permitido profundizar en el análisis de las técnicas de talla para los distintos sitios, se pudo observar en términos generales la presencia de rasgos vinculados tanto a técnicas de talla por percusión directa a mano alzada (talones lisos y

Tabla 2. Materiales líticos recuperados en los sitios arqueológicos del departamento Gualeguay. Entre los ecofactos se cuantifican también rodados de tamaño muy pequeño $(<3 \mathrm{~cm})$ que no son considerados en el análisis descripto en el texto. Estos sábulos han sido identificados como desechos de materia prima (Apolinaire 2017) desprendidos de la matriz de las areniscas y no se registraron evidencias de su talla. Elaborado por Eduardo Apolinaire (2017).

\begin{tabular}{|c|c|c|c|c|c|}
\hline \multirow{2}{*}{ Sitio } & \multicolumn{5}{|c|}{ Clases tipológicas } \\
\hline & Instrumentos & Núcleos & Desechos de talla & Ecofactos & Total \\
\hline CDLI 1 & 0 & 11 & 12 & 5 & 28 \\
\hline CDLI 2 & 6 & 6 & 21 & 23 & 56 \\
\hline PDLL & 0 & 1 & 2 & 3 & 6 \\
\hline LDN 1 & 1 & 2 & 10 & 3 & 16 \\
\hline LDN 2 & 1 & 1 & 1 & 0 & 3 \\
\hline LA & 9 & 5 & 11 & 14 & 39 \\
\hline LCO & 5 & 2 & 14 & 11 & 32 \\
\hline LC 1 & 2 & 8 & 26 & 2 & 38 \\
\hline LC 2 & 2 & 8 & 9 & 3 & 22 \\
\hline EED 1 & 1 & 2 & 10 & 0 & 13 \\
\hline EED 2 & 0 & 0 & 5 & 0 & 5 \\
\hline $\mathrm{TP}$ & 1 & 0 & 0 & 14 & 15 \\
\hline AEP & 0 & 1 & 1 & 3 & 5 \\
\hline MEP 2 & 0 & 1 & 6 & 1 & 8 \\
\hline Total & 28 & 51 & 125 & 82 & 286 \\
\hline
\end{tabular}

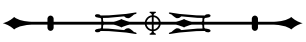



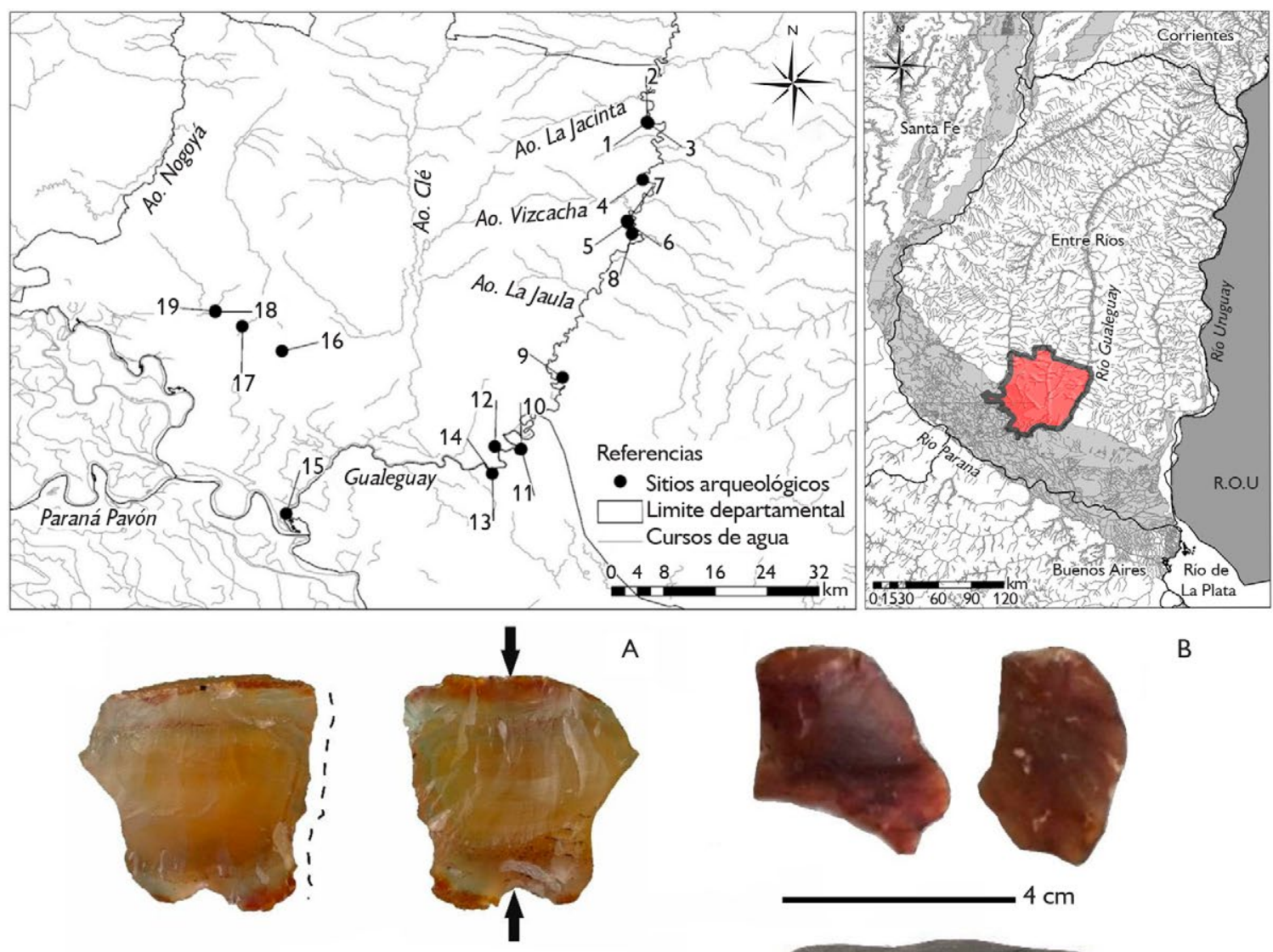

A
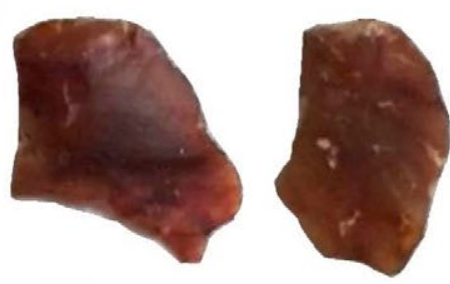

B

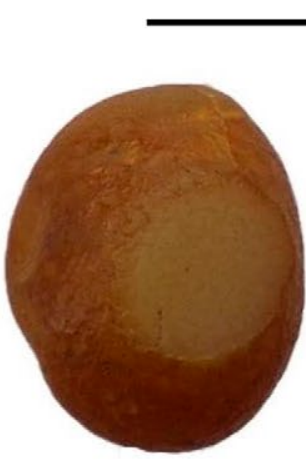

$4 \mathrm{~cm}$

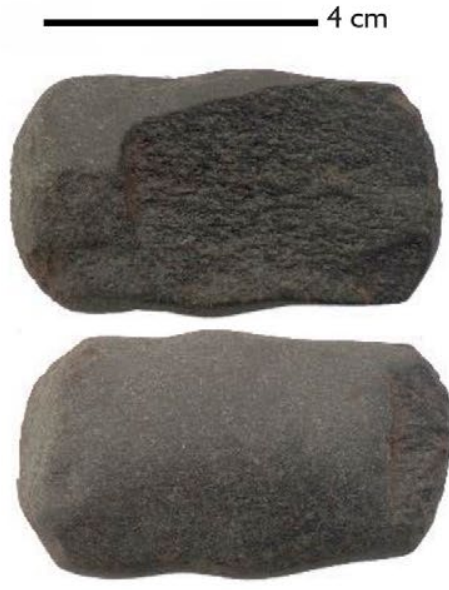

D

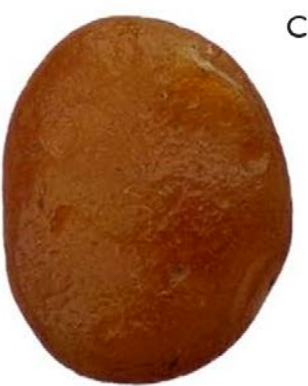

C

$3 \mathrm{~cm}$

Figura 5. Arriba: ubicación de los sitios arqueológicos: 1) Cementerio de los Indios 1 (CDLI 1); 2) Cementerio de los Indios 2 (CDLI 2); 3) Cementerio de los Indios 3 (CDLI 3); 4) Paso de La Lana (PDLL); 5) Laguna del Negro 1 (LDN 1); 6) Laguna del Negro 2 (LDN 2); 7 ) Laguna del Negro 3 (LDN 3); 8) La Amairú (LA); 9) Laguna de Colman (LCO); 10) La Chimenea 1 (LCH 1); 11) La Chimenea 2 (LCH 2); 12) Tres Palos (LCH 3); 13) Estancia El Destino 1 (EED 1); 14) Estancia El Destino 2 (EED 2); 15) Laguna La Nicanora (LLN); 16) Punta del Monte (PDM); 17) Arroyo El Portugués (AEP); 18) Médano El Pencal 1 (MEP 1); 19) Médano El Pencal 2 (MEP 2). Abajo: artefactos sobre rodados fluviales: A) instrumento sobre núcleo bipolar elaborado en calcedonia; B) desechos de talla sobre calcedonia; C) artefacto activo no diferenciado sobre rodado fluvial; D) hacha sobre rodado de basalto. Elaborado por Eduardo Apolinaire (2017).

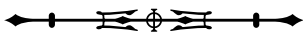


espesos, bulbos prominentes) (Figura 5B) como evidencias de talla bipolar (talones astillados, bulbos difusos, ondas de percusión y estrías) (Figura 5A). La técnica empleada parece relacionarse, en primer lugar, con la materia prima aprovechada y el tipo de depósito de procedencia de las rocas. La técnica bipolar sólo se registró con relación a la talla de rocas criptocristalinas (e.g. calcedonia, caliza silicificada, xilópalo), que mayoritariamente provienen de rodados fluviales. Por otro lado, al considerar únicamente el subconjunto de rocas criptocristalinas, se observa que los atributos asociados a la técnica bipolar son más frecuentes en los sitios septentrionales, en donde se observan núcleos y lascas bipolares y/o desechos de talla con posibles rasgos asociados a esta técnica (i.e. talones astillados, corticales y puntiformes asociados a bulbos difusos o negativos). Estas diferencias han sido interpretadas en función a la variabilidad que estos dos sectores presentan en cuanto a la abundancia de afloramientos de materias primas silicoclásticas (Apolinaire, 2017).

Parte de los nódulos que ingresaron al área (departamento Gualeguay) consisten en rodados fluviales de diversa litología y tamaño. Esto se infiere a partir de la presencia de artefactos sobre rodados en diferentes estadios de reducción, así como de clastos sin modificar. En este último caso, es interesante mencionar que se recuperaron abundantes rodados muy pequeños (de tamaños menores a los $2 \mathrm{~cm}$ ) que podrían corresponder a desechos producidos durante la talla de rocas silicoclásticas. Las areniscas aflorantes en el área presentan clastos tamaños sábulo y gravilla $(<2 \mathrm{~cm})$ incorporados en su esqueleto, los cuales se desprenden fácilmente durante la fractura de las rocas. Otros rodados de tamaños mayores, en cambio, fueron transportados a los sitios desde depósitos secundarios para su posterior uso como materias primas para la elaboración de artefactos.

La muestra considerada a continuación se compone de 206 materiales líticos recuperados en los sitios antes señalados, consistentes en rodados sin modificar y artefactos elaborados sobre rodados; estos últimos conservan una gran parte de la corteza y por tanto del volumen original del nódulo, con lo cual pueden estimarse sus dimensiones de modo relativamente confiable. El eje mayor promedio de los clastos fue estimado en $31 \mathrm{~mm}$ y su eje secundario en $10,5 \mathrm{~mm}$. Si se consideran las categorías de tamaño ${ }^{2}$, una gran parte de la muestra presenta tamaños pequeño $(48,0 \%)$ y mediano pequeño $(40,2 \%)$, si bien también se encuentran representadas las categorías mediano grande $(8,7 \%)$, grande $(2,4 \%)$ y muy grande $(0,4 \%)$.

Entre aquellos artefactos cuya materia prima pudo ser identificada ( $n=144)$, la calcedonia es la roca más abundante $(70,75 \%)$, seguida por la caliza silicificada $(20,23 \%)$; en menores porcentajes se registró cuarcita $(3,47 \%)$, cuarzo $(1,39 \%)$, basaltos $(2,78 \%)$, pizarra $(0,69 \%)$ y xilópalo (0,69\%). Cabe señalar que muchos de los elementos cuya materia prima no pudo establecerse corresponden a rodados sin modificar en los cuales la corteza y/o la falta de una fractura fresca imposibilita su identificación petrográfica. En cuanto al color interno de los materiales considerados, las tonalidades más frecuentes son las rojizas $(68,12 \%)$ seguidas por los colores castaños (14,40\%), blancos y translúcidos (10,19\%), grises (3,40\%), amarillos $(2,43 \%)$ y negros (1,46\%).

Si consideramos las características que se registraron en los depósitos naturales de rodados del río Uruguay inferior observamos que, debido a la pérdida de capacidad de carga del río, en los depósitos más meridionales es altamente improbable encontrar rodados con un eje mayor superior a los $5 \mathrm{~cm}$. Por otra parte, en estos depósitos meridionales la caliza silicificada se presenta en mayores porcentajes que en aquellos ubicados hacia el norte, si bien la calcedonia es la materia prima predominante a lo largo de todos los depósitos. Los artefactos y ecofactos sobre rodados recuperados

\footnotetext{
2 Sensu Aschero (1975).
}

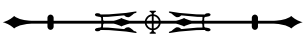


en los sitios del área de estudio presentan tamaños predominantemente pequeño y mediano pequeño. Además, las rocas predominantes en los sitios son las calcedonias y en segundo lugar, la caliza silicificada. Por lo tanto, los tamaños y litologías registradas en los sitios son en general compatibles con las descriptas para los depósitos meridionales, que son los más cercanos al área de estudio. Sin embargo, existen casos particulares que no responden a estas tendencias generales. En el sitio Laguna del Negro 1 (LDN1) se recuperó un hacha elaborada sobre rodado de basalto (Figura 5D) que presenta un módulo de tamaño muy grande. Este instrumento fue elaborado sobre un nódulo que, debido a su tamaño, no pudo ser aprovisionado en los depósitos más meridionales. Además, no se recuperaron en este sitio desechos de talla sobre esta materia prima, con lo cual es probable que este artefacto haya ingresado al área como un instrumento ya finalizado. Una situación similar ha sido propuesta para explicar la presencia de otros instrumentos de impacto recuperados en LDN1 cuyas materias primas (e.g. granitoides y gabroides) proceden de afloramientos primarios alóctonos (Apolinaire, 2017).

Se registraron otras importantes diferencias entre los rodados naturales y los arqueológicos. Una de las más interesantes se relaciona con la distribución de las categorías de color: en los sitios arqueológicos predominan las tonalidades rojizas, mientras que en los depósitos naturales son más frecuentes los rodados castaños y transparentes. Además, es notoria la ausencia en los sitios arqueológicos de rodados de arenisca, si bien esta litología es la tercera categoría en orden de abundancia en los depósitos naturales. Esto último puede deberse a la presencia de afloramientos primarios de arenisca al norte del área de estudio, en las barrancas del arroyo Jacinta. La elevada disponibilidad de esta clase de materia prima a nivel local (al menos para los sitios más septentrionales dentro del área de estudio) puede explicar que las areniscas no hayan sido seleccionadas en los depósitos secundarios.

En suma, a partir de la comparación de las muestras arqueológicas con las características de los depósitos de rodados del río Uruguay inferior, se propone que el abastecimiento de rodados fluviales habría ocurrido principalmente en los depósitos secundarios más meridionales. Estos depósitos de rodados son los más cercanos a los sitios del área de estudio y constituyen una fuente predecible y prácticamente inagotable de materia prima localizada a media distancia ${ }^{3}$. En ellos las elecciones tecnológicas se manifestaron a través de distintos criterios de selección: se recolectaron principalmente rodados criptocristalinos de calcedonia y caliza silicificada y, además, las tonalidades rojizas fueron las más privilegiadas. La selección de tonalidades rojizas se manifiesta también en las cadenas operativas de otras clases de materias primas. Se ha propuesto que esta búsqueda del color rojo condicionaría fuertemente el aprovisionamiento de calizas silicificadas de afloramientos primarios $y$, por otro lado, también podría explicar la termoalteración de areniscas en el área de estudio (Apolinaire, 2017). Como se ha descrito en trabajos previos (Apolinaire et al., 2016; Apolinaire, 2017), es posible afirmar que las areniscas que afloran dentro de la provincia de Entre Ríos (formaciones Ituzaingó y Salto Chico), al ser sometidas a temperaturas inferiores al estrés térmico del cuarzo, cambian rápida y notablemente su color. En cuanto a las propiedades para la talla, se observa una pérdida de la homogeneidad del cemento silíceo y la transformación mineralógica de los minerales de hierro y arcillas. Estos cambios debilitan el cemento y conducen a que la fractura deba rodear los clastos del esqueleto, en lugar de producirse por planos que lo atraviesen. Esto produce un menor control de las fracturas y vuelve a las rocas más quebradizas y deleznables. Por ese motivo, la termoalteración registrada en las muestras arqueológicas posiblemente no se relacione con la intención de mejorar

\footnotetext{
3 Sensu Flegenheimer y Bayón (1999).
}

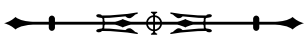


de la calidad de la materia prima, sino que podría ser el resultado de una alteración involuntaria o accidental, o bien, de la búsqueda de alterar otro tipo de propiedades de las rocas (p. ej., color).

\section{CONCLUSIONES}

Los depósitos secundarios de rodados fluviales representan una amplia zona de aprovisionamiento de todas las materias primas aflorantes en el río Uruguay. Estos depósitos componen una estrecha franja sobre la costa del río donde es posible tener un fácil acceso a cantidades prácticamente inagotables de materia prima. A lo largo de esta área de aprovisionamiento, se registraron variaciones en las características de los rodados. En todos los tramos analizados del río se encuentran disponibles las diferentes morfologías de nódulos, si bien existen algunas diferencias menores en cuanto a su representación relativa. En este sentido, la disponibilidad de rodados de diversa morfología no es condicionada geográficamente. En el caso de la litología, se observó que la calcedonia es la materia prima predominante a lo largo de todo el río, seguida por el cuarzo cristalino y la arenisca. La principal diferencia a nivel litológico está dada por el aumento de la caliza silicificada en el tramo meridional, relacionada con la presencia de afloramientos primarios en este sector. Las mayores variaciones geográficas se observan en relación a las dimensiones de los nódulos. En el tramo más septentrional del curso del río se encuentran los depósitos de rodados con mayores volúmenes. A partir de esto es posible concluir que el aprovisionamiento de nódulos mayores es más factible al noreste de la provincia. La pérdida de competencia del río genera depósitos de dimensiones cada vez menores hacia el sur. De hecho, al sur de la localidad de Puerto Campichuelo, no se detectaron depósitos secundarios de rodados. En suma, los depósitos secundarios concentran las rocas que afloran a lo largo del río Uruguay, permitiendo acceder a unidades de materia prima de tamaños reducidos pero de variada morfología y litología.

\section{REFERENCIAS}

ACEÑOLAZA, Florencio Gilberto. Geología y recursos geológicos de la Mesopotamia Argentina. San Miguel de Tucumán: Consejo Nacional de Investigaciones Científicas y Técnicas, Instituto Superior de Correlación Geológica, Universidad Nacional de Tucumán, 2007. (Serie Correlación Geológica, 22).

APOLINAIRE, Eduardo. Arqueología del suroeste de Entre Ríos: tecnología, subsistencia e interacción social en tiempos prehispánicos. 2017. 360 f. Tesis (Doctorado en Ciencias Naturales) - Universidad Nacional de La Plata, La Plata, 2017.

APOLINAIRE, Eduardo; BASTOURRE, Laura; ANGRIZANI, Rodrigo Costa. Arqueología de las tierras altas de Entre Ríos: primeros resultados de las prospecciones en el interior del departamento Gualeguay. Intersecciones en Antropología, Olavarría, v. 17, n. 1, p. 91-107, marzo 2016.

ASCHERO, Carlos. Ensayo para una clasificación morfológica de artefactos líticos aplicada a estudios tipológicos comparativos. Informe presentado al CONICET, Buenos Aires, 1975.

BANEGAS, Anahí; GOYE, María Soledad; GÓMEZ OTERO, Julieta. Caracterización regional de recursos líticos en el nordeste de la provincia del Chubut (Patagonia argentina). Intersecciones en Antropología, Olavarría, v. 16, p. 39-50, oct. 2015. Suplemento 1.

BASTOURRE, Laura; APOLINAIRE, Eduardo. Estudios arqueofaunísticos en un contexto estratigráfico de las llanuras interiores de Entre Ríos: el sitio Laguna del Negro 1 (departamento Gualeguay, Argentina). Boletim do Museu Paraense Emílio Goeldi. Ciências Humanas, Belém, v. 12, n. 2, p. 453-417, maio-ago. 2017.

BAYÓN, Cristina; FLEGENHEIMER, Nora; PUPIO, Alejandra. Planes sociales en el abastecimiento y traslado de roca en la pampa bonaerense en el Holoceno temprano y tardío. Relaciones de la Sociedad Argentina de Antropología, Buenos Aires, v. 31, n. 1, p. 19-45, sem. 2006.

BERÓN, Mónica Alejandra. Base regional de recursos minerales en el occidente pampeano: procedencia y estrategias de aprovisionamiento. Relaciones de la Sociedad Argentina de Antropología, Buenos Aires, v. 3, n. 1, p. 47-88, sem. 2006.

BERÓN, Mónica Alejandra; MIGALE, Laura Alejandra; CURTONI, Rafael Pedro. Hacia la definición de una base regional de recursos líticos en el área del Curacó. Una cantera taller: Puesto Córdoba (La Pampa, Argentina). Relaciones de la Sociedad Argentina de Antropología, Buenos Aires, v. 20, n. 1, p. 111-128, sem. 1995.

BOBILLO, Federico Miguel; HOCSMAN, Salomón. Mucho más que sólo aprovisionamiento lítico: actividades en canteras y prácticas sociales en las fuentes de Pampa Oeste, Quebrada Seca y Punta de la Peña (Antofagasta de la Sierra, Catamarca). Revista del Museo de Antropología, Córdoba, v. 8, n. 1, p. 22-44, jun. 2015.

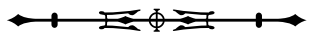


BONOMO, Mariano; PRATES, Luciano. La explotación de depósitos secundarios de rodados en el curso medio del Río Negro y el litoral Marítimo Pampeano. En: ESCOLA, Patricia Susana; HOCSMAN, Salomón (Ed.). Artefactos líticos, movilidad y funcionalidad de sitios: problemas y perspectivas. Oxford: Archaeopress, 2014. p. 77-92. (BAR International Series, n. 2628)

BONOMO, Mariano; BLASI, Adriana Mónica. Base regional de recursos líticos del Delta del Paraná. Estudio petrográfico de artefactos y afloramientos en el sur de Entre Ríos. CazadoresRecolectores del Cono Sur. Revista de Arqueología, Mar del Plata, v. 4, n. 1, p. 17-41, anual 2010.

BUC, Natacha; SILVESTRE, Romina. Distribución de artefactos líticos y óseos en el humedal del Paraná inferior. Relacionando conjuntos del norte y sur del Paraná Guazú. En: COCCO, Gabriel; FEUILLET TERZAGHI, María Rosario (Comp.). Arqueología de cazadores recolectores en la Cuenca del Plata. Santa Fe: Centro de Estudios Hispanoamericanos, 2011. p. 171-188.

CARBALLIDO CALATAYUD, Mariana; PEREYRA, Fernando Xavier. Determinación de la base regional de recursos líticos del área río Manso inferior-Foyel (Río Negro). Primeros resultados. Comechingonia: Revista de Arqueología, Córdoba, v. 16, n. 1 , p. 287-296, sem. 2012.

CASTRO, Juan C. Arqueología del centro de la provincia de Entre Ríos (Argentina). Arqueología, Buenos Aires, v. 19, n. 2 , p. 287-304, cuat. 2013.

COLOMBO, Mariano. Los cazadores recolectores pampeanos y sus rocas: la obtención de materias primas líticas vista desde las canteras arqueológicas del centro de Tandilia. 2013. $360 \mathrm{f}$. Tesis (Doctorado en Ciencias Naturales) - Universidad Nacional de La Plata, La Plata, 2013.

COLOMBO, Mariano; FLEGENHEIMER, Nora. La elección de rocas de colores por los pobladores tempranos de la región Pampeana (Buenos Aires, Argentina): nuevas consideraciones desde las canteras. Boletín del Museo Chileno de Arte Precolombino, Santiago, v. 18, n. 1, p. 125-137, sem. 2013.

COTTERELL, Brian; KAMMINGA, Johan. The formation of flakes. American antiquity, Washington, v. 52, n. 4, p. 675-708, Oct. 1987

ERICSON, Jonathon E. Toward the analysis of lithic production systems. In: ERICSON, Jonathon E.; PURDY, Barbara A. (Ed.). Prehistoric Quarries and Lithic Production. Cambridge: Cambridge University Press, 1984. p. 1-9. (New directions in Archaeology).

FERRERO, Brenda; BRANDONI, Diego; NORIEGA, Jorge I.; CARLINI, Alfredo. Mamíferos de la Formación El Palmar (Pleistoceno tardío) de la provincia de Entre Ríos, Argentina. Revista del Museo Argentino de Ciencias Naturales, Buenos Aires, v. 9, n. 2, p. 109-117, sem. 2007.
FLEGENHEIMER, Nora; BAYON, Cristina. Abastecimiento temprano de rocas en sitios pampeanos tempranos: recolectando colores. En: ASCHERO, Carlos; KORSTANJE, María; VUOTO, Patricia (Org.). En los Tres Reinos: prácticas de recolección en el Cono Sur de América. Tucumán: Instituto de Arqueología y Museo, Facultad de Ciencias Naturales e Instituto Miguel Lillo, 1999. p. 95-107.

FRANCO, Nora; BORRERO, Luis. Metodología de análisis de la estructura regional de recursos líticos. En: ASCHERO, Carlos; KORSTANJE, María; VUOTO, Patricia (Org.). En los Tres Reinos: prácticas de recolección en el Cono Sur de América. Tucumán: Instituto de Arqueología y Museo, Facultad de Ciencias Naturales e Instituto Miguel Lillo, 1999. p. 27-37.

GENTILE, Carlos; RÍMOLDI, Héctor. Mesopotamia. En: SIMPOSIO DE GEOLOGÍA REGIONAL ARGENTINA, 2., 1979, Córdoba. Anales... Academia Nacional de Ciencias de Córdoba: Córdoba, 1979. p. 185-223

GOSO, H.; BOSSI, J. Cenozoic Stratigraphy of the Rio Grande do Sul coastal province. Boletim Paranaense de Geociências, Curitiba, v. 33, n. 1, p. 54-55, sem. 1966.

HEIDER, Guillermo; RIVERO, Diego; BALDO, Edgardo. Rocas de uso arqueológicos en las Sierras Centrales. Fuentes de recursos líticos identificadas y potenciales en las provincias de Córdoba y San Luis, Argentina. Revista del Museo de Entre Ríos, Paraná, ARG, v. 1, n. 2, p. 55-72, sem. 2015.

HERMO, Darío; TERRANOVA, Enrique; MOSQUERA, Bruno; FRUTOS, Julián. Base regional de recursos líticos en la Meseta de Somuncurá: primeros resultados en la cuenca del Arroyo Talagapa (Río Negro). En: ZANGRANDO, Atilio; BARBERENA, Ramiro; GIL, Adolfo; NEME, Gustavo; GIARDINA, Miguel; LUNA, Leandro; OTAOLA, Clara; PAULIDES, Salvador; SALGÁN, Laura; TÍVOLI, Ángela. (Comp.). Tendencias Teórico-Metodológicas y casos de estudio en la arqueología de la Patagonia. San Rafael: Museo de Historia Natural de San Rafael, 2013. p. 109-117.

HOCSMAN, Salomón. Caracterización petrográfica y calidades de areniscas (margen entrerriana del Paraná medio). Revista de Antropología del Museo de Entre Ríos, Paraná, ARG, v. 1, n. 1, p. 1-11, sem. 2015.

IRIONDO, Martín. Geología del Cuaternario en Argentina Corrientes: Editorial Moglia, 2010.

IRIONDO, Martín. El cuaternario de Entre Ríos. Revista de la Asociación de Ciencias Naturales del Litoral, Santa Fe, v. 11, n. 1, p. 125-141, 1980. DOI: https://doi.org/10.14409/natura.v1i11.3375.

IRIONDO, Martín; KRÖHLING, Daniela. Cambios ambientales en la cuenca del Uruguay (desde el presente hasta dos millones de años atrás). Santa Fe: Universidad Nacional del Litoral, 2008.

JONES, Andrew; MACGREGOR, Gavin. Colouring the past: the significance of colour in archaeological research. Oxford: Berg Publishers, 2002.

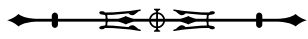


KRÖHLING, Daniela. La Formación El Palmar, una unidad fluvial asignable al subestadio cálido EIO 5A (Pleistoceno Tardío) de la cuenca del río Uruguay. Natura Neotropicalis, Santa Fe, v. 40, n. 1, p. 61-86, anual 2009.

MARTÍNEZ, Sergio; VEROSLAVSKY, Gerardo; CABRERA, Fernanda. Calizas del Queguay: un enfoque hacia la arqueología. Revista de Antropología del Museo de Entre Ríos, Paraná, ARG, v. 1, n. 2, p. 1-10, sept. 2015.

NAMI, Hugo Gabriel. El subsistema tecnológico de la confección de instrumentos líticos y la explotación de los recursos del ambiente: una nueva vía de aproximación. Shincal, Catamarca, v. 2, n. 1, p. 33-53, 1992.

NORIEGA, Jorge I.; CARLINI, Alfredo A.; TONNI, Eduardo P. Vertebrados del Pleistoceno tardío de la cuenca del Arroyo Ensenada (Departamento Diamante, provincia de Entre Ríos). INSUGEO, Miscelánea, Tucumán, v. 12, n. 1, p. 71-76, 2004.

SHEENAN, Stephen. Quantifying Archaeology. Edimburgh: Edimburgh University Press, 1988.

SKARBUN, Fabiana. Estructura y explotación de los recursos líticos en el sector meridional de la meseta central de Santa Cruz, Argentina. Magallania, Punta Arenas, v. 43, n. 1, p. 191-209, mayo 2015.

TAÇON, Paul. Rainbow colour and power among the Waanyi of Northwest Queensland Cambridge Archaeological Journal, Cambridge, v. 18, n. 2, p. 163-176, June 2008. DOI: https://doi. org/10.1017/S0959774308000231.
TONNI, Eduardo P. Stegomastodon platensis (Mammalia, Proboscidea, Gomphotheriidae) y la antigüedad de la Formación El Palmar en el Departamento de Colón, provincia de Entre Ríos, Republica Argentina. Ameghiniana, Buenos Aires, v. 24 n. 3-4, p. 323-324, bimensual 1987.

TURNES, Lucas; QUIROGA, Diego; SANTIAGO, Fernando; NEGRE, Joan; GONZÁLEZ GUILLOT, Mauricio. Primera aproximación al estudio de fuentes secundarias de materias primas (área intercuencas río Avilés - río Chico, Tierra del Fuego, Argentina). Intersecciones en Antropología, Olavarría, v. 17, p. 75-87, jun. 2016. Suplemento 2.

VEROSLAVSKY, Gerardo; UBILLA, Martín. A 'snapshot'of the evolution of the Uruguay River (Del Plata Basin): the Salto depositional sequence (Pleistocene, Uruguay, South America). Quaternary Science Reviews, New York, v. 26, n. 22, p. 29132923, Nov. 2007.

ZAR, Jarrold H. Biostatistical analysis. New York: Prentice Hall, 2010

ZINGG, Theodor. Beitrag zur schotteranalyse: die Schotteranalyse und ihre Anwendung auf die Glattalschotter. Schweizerische Mineralogische und Petrologische Mitteilugen, Bern, v. 15, n. 1, p. 39-140, 1935. 